Euskal ikerketen aldizkaria | Revue d'études basques |

Revista de estudios vascos | Basque studies review

$7 \mid 2002$

Numéro VII

\title{
L'écho des représentations de la femme musulmane en Navarre
}

Yvette Cardaillac-Hermosilla

(2) OpenEdition

Journals

Édition électronique

URL : http://journals.openedition.org/lapurdum/964

DOI : 10.4000/lapurdum.964

ISSN : 1965-0655

Éditeur

IKER

Édition imprimée

Date de publication : 1 octobre 2002

Pagination : 177-183

ISBN : 2-86781-321-2

ISSN : $1273-3830$

Référence électronique

Yvette Cardaillac-Hermosilla, "L'écho des représentations de la femme musulmane en Navarre », Lapurdum [En ligne], 7 | 2002, mis en ligne le 01 juin 2009, consulté le 01 mai 2019. URL : http:// journals.openedition.org/lapurdum/964; DOI : 10.4000/lapurdum.964 


\title{
L'écho des représentations de la femme musulmane en Navarre
}

\author{
Yvette Cardaillac-Hermosilla
}

Université Michel de Montaigne Bordeaux 3

Les représentations artistiques et littéraires de la femme musulmane trouvent leur écho en Navarre même si à priori les passages fugaces des troupes destructrices ne prédisposent pas au développement de l'imaginaire dans ce domaine mais se concentrent plutôt sur l'aspect guerrier par peur de la puissance de l'ennemi séculaire. Cependant quelques manifestations présentent des liens qui n'apparaissent pas au premier abord à cause de ceux, très différents, qui existent entre le monde terrestre et le monde céleste dans les cultures chrétiennes et musulmanes. Pour arriver à les percevoir nous partirons de l'examen de la pyxide de Leyre qui nous mènera à la représentation de la femme musulmane dans la poésie arabo-andalouse pour revenir aux saintes Alodia y Nunilo martyrisées par les musulmans.

Dans les pratiques sociales et culturelles d'Al-Andalous sous domination musulmane, la représentation de la musicienne-chanteuse mérite de retenir notre attention.

Au Xl $\mathrm{I}^{\text {ime }}$ siècle, la pyxide en ivoire destinée au premier fils d'Al-Mansour, $\mathrm{Ab}$ Al-Malik et conservée au monastère de Leyre pendant des siècles, date de 1005. Elle nous apporte sa contribution avec un ensemble raffiné.

La partie frontale se compose de trois médaillons avec une scène centrale représentant un groupe de trois musiciennes' qui jouent l'une de la flûte double, l'autre d'une guitare à six cordes et une dernière de la trompe. L'ensemble peut représenter une grande fête offerte au calife dans le jardin d'un palais à la campagne. Ce qui confirme cette idée ce sont les scènes de chasse du pourtour, les scènes de tournoi, un cavalier sur un éléphant et un dresseur de lions. Dans le médaillon de droite un homme assis sur un coussin représenterait le calife Hixen II et à droite dans une position identique se trouverait $\mathrm{Ab} \mathrm{Al-Malik} \mathrm{lui-}$ même. Ce coffret d'ailleurs serait un cadeau d'accession à la majorité pour un adolescent susceptible de devenir souverain, ce qui n'a pas été le cas, à cause de conflits de palais. Cette pyxide se trouve maintenant au musée de Navarre à Pampelune, elle mesure $220 \times 350 \mathrm{~mm}$ de base et $220 \mathrm{~mm}$ de haut et elle a été faite par Faray avec ses disciples selon le texte qui figure à l'intérieur, le médaillon central étant sculpté par Halir, elle vient de l'atelier de Medina Az Zahara. On considère que c'est l'œuvre la plus importante en ivoire de cet atelier et de cette période. Les silhouettes des femmes sont stylisées et dérivées des prototypes orientaux en médaillon circulaire agrémenté de lobes. Cette

\footnotetext{
' Les spécialistes ont polémiqué sur le sexe car aucune caractéristique particulière ne le détermine mais les textes témoignent du goût des princes pour les orchestres féminins présents à la cour.
} 
esthétique, nous la retrouvons dans les miniatures, les personnages ont très peu de relief, ils se perdent dans le décor végétal ou atauriques pour respecter la modestie du sculpteur, car le seul créateur de toute chose est Allah. Cette représentation artistique illustre des faits historiques.

À la cour de Grenade et de Cordoue des orchestres de musicienneschanteuses animaient les soirées et les Chrétiens du nord adoptèrent cette coutume. Ainsi, Ibn al-kinân qui voyageait fréquemment dans le nord de la Péninsule raconte qu'il assista à la réception de la chrétienne fille de Sancho, roi des Basques, épouse du tyran Sancho fils de García, fils de Ferdinand où dansaient un certain nombre de danseuses chanteuses qui lui avaient été offertes par Sulayman Ibn al-Hakam lorsqu'il était prince des croyants à Cordoue. L'une d'elle chanta à la perfection en s'accompagnant d'un luth. De jeunes servantes captives et très belles se trouvaient près d'elle "on eût cru que c'était des quartiers de lune"2.

$\mathrm{Au}$ temps des Omeyyades les princes et les aristocrates faisaient venir d'Orient les esclaves-musiciennes instruites dans l'art du chant et de la danse. Trois médinoises Fadl, Alan et Qalam partagèrent la couche de l'émir Abd alRahman II et lui donnèrent un fils. Elles dirigeaient des orchestres de musiciennes-esclaves pour lesquelles il fit édifier un pavillon spécial. Elles jouaient au cours de fêtes nocturnes et donnaient des spectacles de chants et de danses. Ainsi vint à Séville d'Orient la chanteuse bagdadienne Qamar (XI ${ }^{\mathrm{emc}}$ siècle). On en forma même sur place en Espagne. Au XI ${ }^{\mathrm{emc}}$ siècle l'une d'entre elles écrivait parfaitement, possédait une diction pure, elle excellait dans le maniement des armes et dans l'art de la prestidigitation. Elles, seulement, participaient aux réceptions de l'aristocratie et se mêlaient aux hommes au cours des banquets car la femme mariée isolée du clan des hommes ne devait pas laisser voir son visage aux étrangers.

La littérature et la poésie nous donnent une autre représentation de la femme qui présente des connexions avec les conceptions développées par les troubadours, mais aussi avec la philosophie du carpe diem.

La poésie est le genre noble cultivé par des poètes de cour mais aussi elle est utilisée en forme de chant comme genre très populaire en particulier le zejel ${ }^{3}$ ou hymne sonore dont voici une strophe se référant à la femme :

2 Peres, H., La poésie andalouse en arabe classique au X7ème siècle, Paris, 1953, p. 386-387. Sancho García régna de 995 à 1017. Arié Rachel, "Aspects de l'Espagne musulmane, histoire et culture" in De l'archéologie à l'histoire, Paris, de Boccard, 1997.

3 A partir des jarchas en langue vulgaire Mohamad Ibn Mahmud l'aveugle de Cabra crée les moaxajas ou poèmes strophiques qui sont des chansons en dialecte accessibles à tous publics et Ibn Bayya de Saragosse (mort en 1138) avec l'aide d'expertes esclaves unit le chant chrétien et le chant oriental dans le zejel qui est le fruit de l'hybridisme de la tradition arabe et romane.

La moxaja est apparue à la fin du I $X^{\text {ème }}$ et au début du $X^{\text {ème }}$ siècle. Cette poésie strophique gravite autour d'une copla romance qui est mise dans la bouche d'une femme et dont le ton contraste avec le raffinement courtois des vers qui précèdent. Le contraste stylistique et linguistique entre la partie classique du poème et la jarcha en romance ou en arabe vulgaire crée une dualité pleine d'ironie et d'humour.

En Espagne la littérature hispano-musulmane a développé divers genres : la muwaschaha qui comprend trois strophes de trois vers mono rime, nouveauté en langue arabe développée particulièrement dans l'Espagne musulmane. Elle était généralement écrite en arabe littéraire par des lettrés hommes de cour et elle chantait l'amour tandis que le zejel était écrit en arabe populaire avec le même système. La casida est un poème de 20 à 100 vers de même mètre et de rimes communes.

Les muwaschahas andalouses se propagent moins facilement et finissent par des vers en langue latine ou en castillan. Souvent les copistes ne les reproduisaient pas ou alors avec beaucoup d'erreurs qui les rendent difficilement compréhensibles. Elles fleurirent surtout entre le XI ${ }^{\mathrm{em}} \mathrm{ei}$ et le XIII ${ }^{\text {eme }}$ siècle. Les jarchas étaient des poèmes de genre oral et de style colloquial. On en fit des anthologies en aljamia pendant le XIII ${ }^{\text {eme }}$ siècle. 


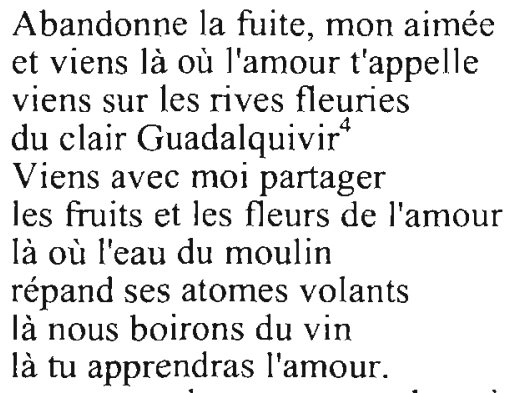

Nous retrouvons dans ce genre de poème chanté le thème de l'amour qui ici suit le carpe diem et l'engouement pour la nature et l'eau toujours présent dans une civilisation issue des déserts d'Arabie autant dans les miniatures que dans la littérature. Nous mentionnerons aussi une muwaschaha appelée aussi chant de la ceinture inventé au IX ${ }^{\text {eme }}$ siècle :

En parcourant les jardins

la muse belle s'amuse ;

sur sa joue fraîche

j'ai posé mes lèvres ardentes ${ }^{5}$.

Ce même désir ardent de jouir du temps présent s'éloigne de l'amour courtois qui s'est longuement développé chez les poètes du désert d'Arabie et qui a pénétré en Espagne avec la poésie venue d'Orient.

Nous n'essaierons même pas d'aborder la polémique qui présente la poésie arabe comme l'une des sources de l'amour chanté par les troubadours. Cependant les divers échanges constatés entre Orient et Occident sur la Péninsule nous permettent d'accepter cette idée parfois décriée.

Mais en ce qui concerne la représentation de la fermme musulmane elle ne concerne ici qu'une minorité : la musicienne-chanteuse n'est ni une femme libre ni une femme esclave ; elle a un statut tout à fait privilégié. Elle est là pour le plaisir de la cour, du sultan à cause de sa beauté et à cause de sa culture. Les poésies d'amour peuvent la concerner mais aussi se référer à d'autres femmes ayant un statut privilégié comme la princesse Wallada poétesse aux mœurs libres assez mal considérées par la société musulmane conservatrice. Fille du calife Omeyyade al-Mustakafi, elle menait une existence de femme libre se jouant des scrupules de la morale instaurée, elle n'ignorait rien des belles manières ni des lois de la poésie qu'elle pratiquait. Elle brodait des vers d'amour sur ses vêtements.

Umm al-Kairam fille du monarque d'Alméria al-Mutasim s'éprit d'un jeune homme de Denia appelé le maréchal-ferrant, l'amour brisant les barrières sociales :

A l'heure où les ombres de la nuit seront obscures, tu attendras ma visite ; secret.

car pour moi la nuit est ce qu'il y a de plus propice pour cacher mon lune,

Comme tu m'as fascinée! Si ton charme ensorceleur s'exerçait sur la

${ }^{4}$ Deja mi bien el huir, / Y ven do amor te convida ; / ven a la margen florida / del claro Guadalquivir ; / ven conmigo a compartir / de amor el fruto y las flores, / do en atomos voladores / esparce el agua el molino ; / allí beberemos vino, / allí aprenderás amores.

${ }^{5}$ Recorriendo los jardines / linda musa se divierte ; / sobre su fresca mejilla /pose mis labios ardientes, /y dije : ; bendito sea /el punto en que logro verte ! / antes que la vida nos haya dejado, /del goce apuremos el vaso encantado. ibid p. 252. 
elle n'apparaîtrait plus ;

sur la nuit, elle ne couvrirait plus la terre de son ombre et sur l'étoile, elle ne voyagerait plus dans le firmament ${ }^{6}$.

Dans la poésie à l'époque Almohade (à partir de 1146) il faut remarquer le développement de la poésie féminine qui jusqu'alors était représentée par des poèmes occasionnels attribués à un personnage. Il semblerait que cette présence ait ses origines dans la tradition matriarcale de certains groupes berbères bien représentés dans la dynastie Almohade. L'un des noms qui se détache parmi ceux des poétesses est celui de Hafsa Bint Al-Hayy Al-Rakuniya de Grenade d'origine berbère et qui jouit d'une liberté insolite pour son temps jusqu'au point que la plus grande partie de ses créations sont destinées à son amant Abu Ya'far Ibn Said. Les souverains, malgré la rigueur religieuse régnante, l'amènent à Marrakech comme préceptrice des fillettes de la famille royale après avoir fait exécuter son amoureux qui conspirait contre la dynastie. Cette poésie médiévale féminine arabe est affectée par la même problématique littéraire que celle des autres femmes : elles utilisent les conventions littéraires masculines pour exprimer des sentiments féminins. Ainsi, Hafsa invertit les images de la poésie masculine et utilise les topiques de la description de la beauté féminine en se les appliquant lorsqu'elle écrit à l'homme qu'elle aime pour l'avertir de sa visite :

Une visite arrive chez to $i^{7}$

avec son cou de gazelle, croissant de lune dans la nuit

son regard a tout le charme de Babylone ${ }^{8}$.

La poésie est considérée comme la reine des arts souvent c'est une poésie de cour et les rois et les courtisans sont poètes et il est de bon ton de se montrer amoureux dans un jardin qui est la représentation du paradis d'Allah, la femme étant la huri que l'on décrit dans les panégyriques floraux comme celui d'Ibn Darray poète d'Al Mansour $\left(n^{\circ} 21\right)$ où la description de la fleur sert de prélude à l'éloge' :

Son sourire est un lys dressé ${ }^{10}$.

Le portrait de la femme andalouse nous est donné par les vers composés au $\mathrm{XI}^{\mathrm{èmc}}$ siècle. Ibn Zaydun écrit :

"Pudique, elle dissimule son visage derrière ses mains / lorsqu'elle le découvre, son corps comparé à celui d'une gazelle, / ses joues font penser au beau jardin parfumé et aux roses, / les dents fines à des perles alignées."

L'idéal classique est représenté par la brune à chevelure luxuriante mais le poète rêve aussi d'une blonde aux cheveux courts. La femme au cou de cygne inspire les poètes.

Tbn Hazm a un faible pour les femmes blondes, à l'image des omeyyades d'Espagne. Au XIV ${ }^{\text {eme }}$ siècle, Ibn al-Jatib voit ainsi les femmes de Grenade:

-I a culture intellectuelle des femmes musulmanes (Garulo, T., Diwan).

Arié, Rachel "Aperçus sur la femme dans l'Espagne musulmane", in Aspects de lEspagne musulmane, bistoire et culture, Paris de Broccard, 1997. Poème de Wallada (princesse) à Ibn Zaydun (vizir)

7 Rubiera Mata, María Jesús, "Las bellas letras", in Historia de Lispaña, el retroceso territorial de Al Andalus, Madrid, Espasa Calpe, 1988, p. 607-634.

8 Una visitante llega a tu casa : su cuello es de gacela, : es una luna creciente sobre la noche ; : su mirada tene el cmbrujo de Babilonia : y su saliva us inejor que : el hijo de las parras, : sus mejillas afrentan a las rosas : y sus dientes confunden a las perlas : ¿ Puede pasar con tu permiso, : o a de irse por alguna circunstancia?

9 "Manifestaciones artísticas" in Historia de España de Menéndes Pidal, Ramón, t. 8, dingé par José Maria Jover Zamora, Madrid, Espasa Calpe, 1994, p. 589-643, p. 602.

10 Si sonrie el rostro de la primavera / es la erguida azucena su sonrisa, / hermosa y riente boca, desaliva / perfumada con el alierto de la amada 
"caractérisée par un embonpoint modéré, des formes voluptueuses et de longues chevelures... elles ont la bouche saine et elles exhalent une bonne odeur. Elles sont vives, parlent avec élégance et ont une conversation agréable. Cependant, les femmes de grande taille parmi elles sont rares".

Les hommes et les femmes se distinguaient par la coiffure, les hommes coiffés de feutre et les femmes portant une pièce d'étoffe. Elles portent des escarpins noirs à bout recourbé d'un emploi courant en Castille au XIII ${ }^{\mathrm{ème}}$.

A partir du XII ${ }^{\text {emc }}$ siècle les femmes andalouses n'ont pas vraiment respecté la pratique du port du voile sur le visage. Au milieu du XIVème siècle, des femmes dévoilées se pressaient avec les hommes sur le passage du cortège royal. Levy-Provencal remarque que la femme andalouse jouit d'une relative libcrté de mouvement dans les couches moyennes de ia population. La femme de la campagne ou la femme artisan travaille sans voile ou la tête couverte d'un voile laissant le visage à découvert ${ }^{11}$. La condition de la citadine andalouse s'est améliorée avec la disparition progressive de la polygamie sous l'influence des cours berbères du XI ${ }^{\text {eme }}$ siècle puis des almoravides parmi lesquels les survivances d'un régime de matriarcat ont permis à la femme d'occuper une place prépondérante dans le milieu familial et dans l'appareil social ${ }^{12}$.

L'époque de splendeur de l'art et de la littérature musulmane se développe entre le XI ${ }^{\text {ème }}$ et le XIII ${ }^{\text {èe }}$ siècle mais ellel suppose des implications avec l'art et l'imaginaire chrétien beaucoup moins élaboré pendant cette période dominée par les rapports d'opposition de la reconquête mais aussi par des relations d'admiration et de négociation. Dans ce contexte complexe la femme chanteuse, musicienne, objet d'amour, ou qu'elle soit louée par l'homme ou par la femme reste l'exception bien éloignée du statut de la majorité de la population même si celui qui lui est réservé sur la Péninsule est assez différent de celui du reste du monde musulman à cause du mélange des populations berbères et autochtones comme nous pouvons l'observer dans Le collier de la colombe $e^{13}$.

La femme musulmane et la poésie qu'elle chante ou qui la chante est ici vue de l'intérieur car pour elle en milieu musulman la situation et le regard de l'intérieur vers l'extérieur ou de l'autre sur son identité vont être déterminants dans les diverses représentations données par la littérature ou l'iconographie des Vieux-chrétiens.

Cependant chanteuse ou poétesse, femme aimante ou femme-objet, minorité dans la minorité, elle n'exprime pas la difficulté d'être femme dans le monde islamique et dans une société patriarchale parce qu'elle bénéficie d'un statut privilégié qui la place bien au-dessus de ses congénères. Elle fait partie de l'élite ou elle est en relation avec elle. La masse des femmes ne laisse pas trace de son existence.

En milieu chrétien, la pyxide de Leyre qui par ses représentations nous a conduit à la poésie musulmane sur les femmes et par les femmes a renfermé les reliques des saintes Nunilo et Alodia vénérées à Leyre qui figurent sculptées sur le portail d'entrée de l'église. Leur représentation stylisée et peu élaborée dans ce monastère roman ne présente pas d'intérêt particulier pour une étude iconographique. C'est certainement plus intéressant sur le plan littéraire : la légende fait apparaître le point de vue chrétien. En l'an 848 deux jeunes filles de la bonne société vivaient à Adahuesca dans la province de Huesca. Elles sont filles d'un père musulman et: d'une mère chrétienne ; après le veuvage, cette

\footnotetext{
${ }^{11}$ Levy-Provencal, E., Histoire de l'Espagne musulmane,

${ }^{12}$ Levy-Provencal, E., Histoire de l'Espagne musulmane, t. 3, p. 402-403

${ }^{13} \mathrm{Jbn}$ Haz de Cordóba, el collar de la paloma, Barcelona, Círculo de lectores, 1997.
} 
dernière se remarie avec un fanatique qui s'oppose aux pratiques de la religion chrétienne de ses belles-filles. Elles partent vivre avec leur grand-mère mais on remarque leur conduite qui ne respecte pas la loi coranique. Elles sont accusées devant le juge qui essaye de les faire abjurer en échange de richesses et d'un mariage avantageux. Mais comme elles résistent vaillamment elles sont confiées à des femmes qui doivent leur enseigner la religion musulmane. Devant leur obstination, on les conduit sur la place publique où elles servent d'amusement au peuple avant d'être décapitées. Leurs corps sont exposés pour être dévorés par les oiseaux de proie afin qu'ils ne soient pas enterrés par les chrétiens. Finalement leurs restes sont mis dans une fosse et ils opérèrent de nombreux miracles. ${ }^{14}$ C'est à la demande de la reine Oneca que leurs corps furent transportés au monastère de Leyre qui était dirigé par le père abbé Fortún. À partir de ce moment-là elles ont reçu un culte qui va se confondre avec le prestige du monastère. Une partie des restes fut déposée dans le coffret d'ivoire dont nous avons parlé. Rappelons aussi que le monastère a servi de sentinelle face à l'armée arabe, ce qui lui vaudra la haine d'Almanzor qui, à la fin du $X^{e m c}$ siècle, entreprend plus de cinquante expéditions contre les chrétiens en moins de cinq ans. Le monastère est alors incendié puis reconstruit. Il devient le centre spirituel de toute la région avec Fortún Garcés, dernier roi de sa dynastie qui y prend l'habit après avoir renoncé à la couronne ${ }^{15}$.

Le culte des saintes martyres Nunilo et Alodia ne s'est pas limité à l'Aragon ou à la Navarre mais s'est diffusé à la Rioja et l'Andalousie. Á cause de la sécheresse on effectue un pèlerinage à Leyre. On organise une procession près de la fontaine des saintes avec prières, litanies et chants liturgiques, le prieur sort un os du coffret en ivoire et l'introduit dans la fontaine et lorsqu'il le recueille dans un linge il est baigné de gouttes de sang. Après ce miracle, les récoltes sont bonnes et l'on édifie un retable en l'honneur des Vierges (XVII ${ }^{\text {èc }}$ siècle). Depuis lors, pour commémorer le fait, les pèlerinages ont lieu le 18 avril qui rappellent aussi le transport des reliques de Adahuesca au monastère ${ }^{16}$.

Dans le retable, les saintes en bois sculptées doré suivent les canons de beauté helléniques mais leurs tuniques et leurs manteaux n'ont rien de caractéristique du monde musulman, les livres qu'elles soutiennent avec leurs mains portent l'inscription en latin des paroles de Nunila : Quis separabit nos a caritate Christe cristi. Ainsi que de celles d'Alodia : Certe soror, neque mors, neque vita, neque instantia. Mais dans la scène du martyr des saintes, à leur droite apparaît un musulman avec son cimeterre qui vient d'accomplir sa besogne.

La relation de la sainteté féminine avec le Maure est fréquente dans la Péninsule. Dans l'histoire de la fille du roi maure de Tolède ${ }^{17}$, Casilda fille du roi maure Almenón de Tolède reçoit son initiation au christianisme par une esclave et guérit miraculeusement, elle s'adonne à une vie de sainteté dans un ermitage solitaire près de Briviesca.

Si du point de vue musulman, la musicienne, la poétesse renvoient à la perfection féminine, si elle s'accompagne de jeunesse et de beauté, du point de vue chrétien, la bonne musulmane est celle qui se convertit et meurt pour la foi chrétienne. Les paradoxes de l'histoire font que les reliques seront conservées pendant des siècles dans un coffret d'ivoire musulman représentant les

\footnotetext{
14 Moral Contreras, El monasterio de Sar Suhudor de Lyre, Madrid l.cón, Editorial Livest, 1994, p. 10.

15 Ibid, p. 12

16 Ibid, p. 29

17 Trueba, Cuentos populares, Toulouse, Paris, Privat, 1951,190 p, p. 61-68.
} 
raffinements d'une fête princière et gardant autour de son fermoir central la représentation de la musicienne danseuse qui assurait les délices de la cour califale. D'ailleurs, les patios des palais avec leur fontaine centrale et les quatre ruisseaux qui assurent l'écoulement des eaux sont une image terrestre de la vision céleste.

Les représentations de la sainteté chrétienne d'origine musulmane peuplent le monastère roman. Leurs interventions miraculeuses essaient de faciliter la vie des humains dans cette vallée de larmes selon la vision biblique de l'Ecclésiaste. Mais les figures passent par la stylisation romane puis par les restrictions du concile de Trente qui souhaite supprimer ornements et détails superflus. Ces modèles ont servi aux moralistes et aux érudits pour la construction de l'histoire différenciée du masculin et du féminin qui joue un rôle fondamental dans le développenent de la culture orientale et occidentale. À Leyre, ìa sainteté chrétienne rejoint les représentations du paradis musulman où les houris aux yeux clairs doivent ravir l'éternité des croyants. 